\title{
Experimental study on the method and performance for heat transfer of jet impingement
}

\author{
Bingtao Wang ${ }^{1, a}$, Yongchang Chen ${ }^{1, b}$ \\ ${ }^{1}$ College of Environmental and Energy Engineering, Beijing University of Technology, China \\ a18810327233@163.com, ${ }^{\text {b chenyongchang@bjut.edu.cn }}$
}

\begin{abstract}
Keywords: heat transfer, jet impingement, thermal properties, experimental method
Abstract. A new kind of experimental equipment has been established to prepare for making a study on the heat transfer of jet impingement when quaternary mixed nitrate will be regarded as working fluid. In order to verify the validation of experimental equipment and method, a experimental study on the heat transfer of jet impingement using the water has been carried out, whose results also have been achieved. The results at the stagnation point showed that good consistences for water are found between the current results and some results from classical correlation, which indicate the accuracy and reliability of experimental equipment and method. Meanwhile, the influence of temperature for heat transfer characteristics of stagnation has been made when the temperature is changed, whose results show that thermophysical properties of working fluid have a enormous influence on the Heat transfer performance. The experimental results in this paper provide a necessary technical guarantee for the study of heat transfer of jet impingement utilizing the high temperature molten salt.
\end{abstract}

\section{Introduction}

Heat transfer of jet impingement is a way that can generate a strong effect on heat exchange with the surface in the local area. For the characteristics of short range and thin boundary layer of jet impingement, it can perform a better consequent in the stagnation point when it is compared to the conventional heat transfer. Therefore, it has been widely used in industrial fields, especially in the fields of aerospace, turbines, local cooling of the gas turbine electronic products and so on. Therefore, a lot of researches about the influences from structure of jet and working fluid for heat transfer have been made, especially when the water is regarded as the working fluid. The heat transfer characteristics of water under the circumstances of low temperature below $40^{\circ} \mathrm{C}$ have been given out by Liu [1] through carrying out a large number of experimental studies, and a classical correlation at stagnation point has been given out. Ma \& Zhao [2] also have given out a heat transfer correlation at the stagnation by combining the theoretical calculation with experimental method with seven coolants under the condition of lower temperature. However, the current researches about heat transfer of jet impingement mainly focus on the lower temperature range from $4^{\circ} \mathrm{C}$ to $40^{\circ} \mathrm{C}$ [3] and the type of working fluid [4]. Besides, it is rare to make a series of researches about the heat transfer characteristics of jet impingement at the higher temperature especially when the temperature is above $100^{\circ} \mathrm{C}$. It's pointed out by Yang [7] that thermal properties of fluids play a very important role in the aspect of convective heat transfer. As for thermal properties, it will change along with variety of temperature. With the technology of jet impingement widely used, the effect of temperature on the thermophysical properties of the working fluid is becoming more and more obvious. Therefore, making a profound research about the medium of high temperature is very significant and instructive in practical applications, especially taking the molten salt as the working fluid of jet impingement.

Although the molten salt [8] has been widely used in the field of solar thermal power generation for the reason of its high temperature resistance, low vapor pressure, low viscosity and excellent electrical conductivity, it has not been published about utilizing the molten salt as working fluid of jet impingement. Thus, making connection between molten salt and heat transfer of jet impingement has an enormous practical significance.

In order to ensure the validation of experimental equipment and prepare for making a study on the heat transfer of jet impingement when quaternary mixed nitrate is regarded as working fluid, a 
experimental research, utilizing water being in higher temperature, forcing on heat transfer characters at stagnation point and on the radial distribution, has been made in this paper.

\section{Experimental apparatus and measuring method}

Experimental apparatus. The schematic diagram of this experimental apparatus for jet impingement is shown in Fig. 1. The apparatus consists of two heating systems, a simulated heating device, a data collecting system, a flow measurement system, a insulation system, and a pipeline auxiliary system. The distilled water and quaternary mixed nitrate as the jet fluid are supplied by the pump. One heating system installed in the storage tank is used to make sure to achieve constant temperature flow at the nozzle exit, and the other heating system installed along with pipeline will be utilized when quaternary mixed nitrate is looked as the working fluid. The simulated heating device is made with a constantan film, which provides a constant heat flow $(q)$ by means of a DC voltage device. The data collecting system includes a computer, an Agilent data acquisition instrument, some thermocouples, some Digital Analog Converter and so on. The flow measurement system includes a metal rotor flow meter and a flow measurement box. In order to reduce the heat radiation of the pipeline system to the environment, the compound silicate cotton is laid on the outside of the pipeline system to be used as the heat insulating material to reduce the heat loss of the system.

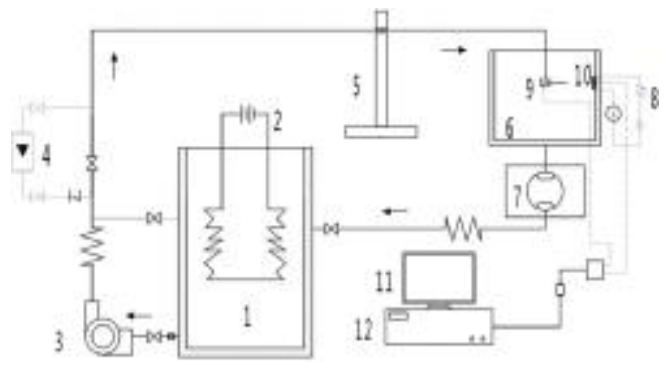

Fig. 1 Schematic diagram of this experimental the apparatus for jet impingement

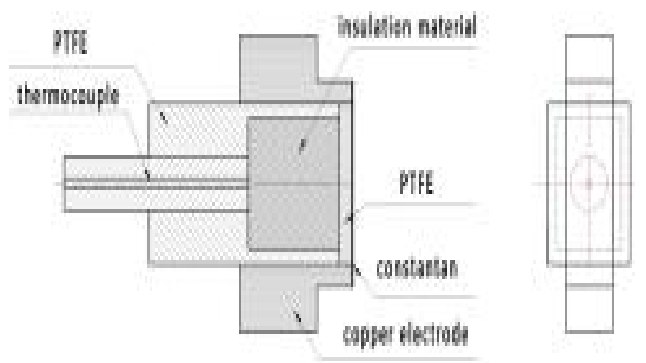

Fig. 2 Schematic diagram of the test section for jet impingement

1.fluid reservoir, 2. electric heater, 3. gear pump, 4. flow meter, 5. adjustable coordinate frame, 6. box, 7. flow-metering device, 8. DC power, 9. nozzle, 10. test section, 11. computer, 12. data collector.

The schematic diagram of the test section used to simulate a heating device is shown in Fig. 2. The size of constantan film is trimmed $20 \mathrm{~mm}$ in length, $8 \mathrm{~mm}$ in weight and $10 \mathrm{um}$ in thickness. Meanwhile, a stainless steel round nozzle $2 \mathrm{~mm}$ in diameter is decided with the distance of z/d realized by adjusting a 3D coordinate frame with an accuracy of $0.01 \mathrm{~mm}$.

Computational method. In order to ensure accuracy of measurement, those high-precision copper-constantan thermocouples utilized in this experiment are calibrated through a platinum resistance with a measured precision of $0.05^{\circ} \mathrm{C}$. It's well known that the heat transfer coefficient for jet impingement can be achieved precisely according to the Newton's law of cooling. Meanwhile, the local heat transfer coefficient at any point can be computed as follows:

$$
h=\frac{q}{t_{w}-t_{f}}
$$

Where the $t_{w}$ is the temperatures of jet target and the $t_{f}$ is the temperature of jet fluid, respectively. As for the heat flow $q$, it can be obtained via dividing the area of constantan film A from heat generation with the equation shown as follows:

$$
q=\frac{I^{2} R}{A}
$$

Meanwhile, The generated heat flux in the process of calculation is controlled by the variation of the output voltage through keeping the current constant and calculated by the current $(I)$ and 
resistance $(R)$ of the constantan film. However, it is worth noting that $R$ can be calculated by the current and voltage of constantan film.

The jet velocity could be calculated by the diameter of nozzle and the volume flux $Q_{V}$. The Reynolds number is calculated on the basis of the hydraulic diameter and the properties of fluid at jet temperature. Thus, the Reynolds number is defined as follows:

$$
R e=\frac{u \cdot d}{v}=\frac{4 Q_{v}}{\pi v d}
$$

The local Nusselt number $(\mathrm{Nu})$ is defined as follows:

$$
N u=\frac{h \cdot d}{\lambda}=\frac{q \cdot d}{\lambda\left(t_{w}-t_{f}\right)}
$$

\section{Experimental results and discussion}

Verification of experimental results. In order to explore the effect of high temperature on the experimental system, the reliability of this experimental system was verified by using $55^{\circ} \mathrm{C}$ water as the jet working fluid at first in this section. The concrete experimental results are made a comparison with the experimental and theoretical correlations given by the Refs with jet impingement of water and are shown in Fig. 3 when the nozzle-to-film distance is in the state of $\mathrm{z} / d=1$.

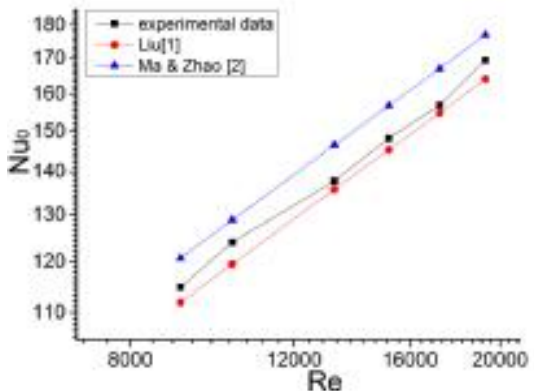

Fig. 3: comparisons of experimental results with literature at stagnation point

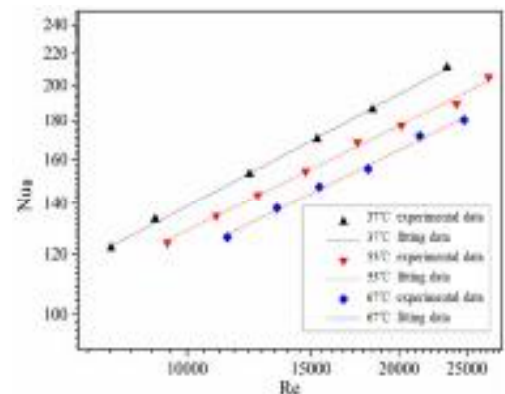

Fig. 4 Stagnation Nusselt number versus Reynolds number for mutative temperature

Fig. 3 shows the comparisons between this work and previous experimental results. According to the Fig. 3, it can be seen obviously that the experimental results of this work are exactly consistent with the previous experimental consequences given by the Ref. [1,2] on the tendency. For the purpose of making a precise contradistinction on the quantitative research, a fitting correlation including the Nusselt number, Reynolds number and Prandtl number at the stagnation for this work is built, whose result is shown as:

$$
N u=0.8515 \cdot \operatorname{Re}^{0.49552} \cdot \operatorname{Pr}^{1 / 3} \quad(8000 \leqq \mathrm{Re} \leqq 22000)
$$

However, at the stagnation, the correlation which is obtained through experiment carried out by Liu[1] is shown as:

$$
N u=0.797 \cdot \operatorname{Re}^{0.5} \cdot \operatorname{Pr}^{1 / 3}, \quad \operatorname{Pr}>3
$$

While the correlation is obtained through theoretical deduction and experiment carried out by Ma $\&$ Zhao[2] is shown as :

$$
N u=0.8598 \cdot \operatorname{Re}^{0.5} \cdot \operatorname{Pr}^{1 / 3}, \quad \operatorname{Pr}>1
$$

Seen from these correlations above, the results of this paper are consistent with the previous correlation with a error from index of Reynolds number being less than $1 \%$, which is consistent with the basic theory of jet impingement heat transfer. Therefore, the present experimental results can be able to ensure the accuracy and reliability of the measurement. 
Effect of temperature on heat transfer of stagnation point. In order to make a further exploration about influence of higher temperature on heat transfer performance, the heat transfer characteristics of water at $37^{\circ} \mathrm{C}, 55^{\circ} \mathrm{C}$ and $67^{\circ} \mathrm{C}$ were measured respectively. As for the physical properties of water at different temperatures, they could be obtained by literature [7]. For the purpose of indicating the influence from temperature on heat transfer, a comparison, for three different qualitative temperatures, forcing on the heat transfer characteristics of stagnation point, has been established and shown in Fig. 4.

For mutative temperatures, the results about the variation of Nusselt number with the increasing Reynolds number are shown in Fig. 4. With the increase of temperature, the effect of heat transfer at stagnation point is becoming increasingly decline under the condition of same Reynolds number and has a same trends of evolution at the stagnation point with increase of Reynolds number. As for the reason of this phenomenon, it is attributed to the decreased Prandtl number because of the increasing temperature. In order to make a precise contradistinction on the quantitative research, some fitting correlations for different temperatures are built with the concrete format shown as:

$$
\begin{array}{ll}
N u=0.9381 \cdot \operatorname{Re}^{0.488} \cdot \operatorname{Pr}^{1 / 3} & 37^{\circ} \mathrm{C} \\
N u=0.8515 \cdot R e^{0.49552} \cdot \operatorname{Pr}^{1 / 3} & 55^{\circ} \mathrm{C} \\
N u=1.3016 \cdot \operatorname{Re}^{0.455} \cdot \operatorname{Pr}^{1 / 3} & 67^{\circ} \mathrm{C}
\end{array}
$$

Comparing these correlations above, it can be found that the index of Reynolds number will range from 0.488 to 0.49552 firstly, and then to 0.455 with the increase of temperature, while these coefficients of correlations will range from 0.9381 to 0.8515 , and then to 1.3016. However, the maximum difference comparing with the classical correlation for index of Reynolds number is less than $9 \%$, which is consistent with the basic theory of jet impingement heat transfer. For the reasons, multiple factors including the viscosity, thermal diffusion coefficient, velocity of fluid and so on may contribute to this effect of heat transfer.

Effect of temperature on radial distribution of heat transfer. The effect of higher temperature on radial distribution of local Nusselt number for water at $\mathrm{z} / \mathrm{d}=1$ is shown in Fig. 5. It can be seen from the Fig. 5 that the radial distributions of local Nusselt number are in a condition of similarity when the temperature ranges from $37^{\circ} \mathrm{C}$ to $67^{\circ} \mathrm{C}$. There is no much difference for radial heat transfer distribution at the outside distance of $\mathrm{r} / \mathrm{d}=1.5$ when the temperature changes. However, a obvious difference takes place at the inner area of $\mathrm{r} / \mathrm{d}=1.5$ for the heat transfer with the increasing temperature. Thus, the change of temperature has no little effect on the characteristics of heat transfer at the stagnation region. In order to illustrate the influence of temperature on the distribution of average Nusselt number, the average Nusselt number is normalized and the results is also shown in Fig. 6.

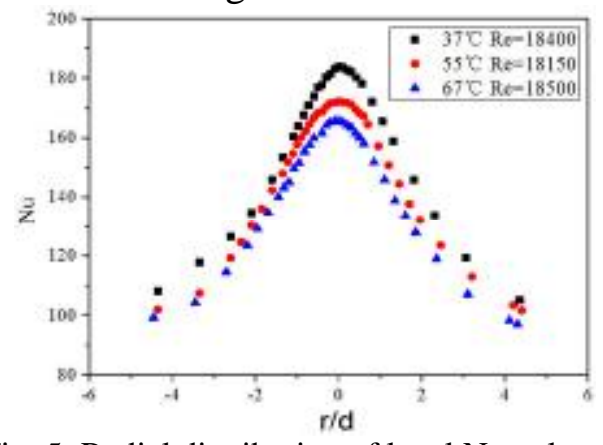

Fig. 5: Radial distribution of local Nusselt number for mutative temperature

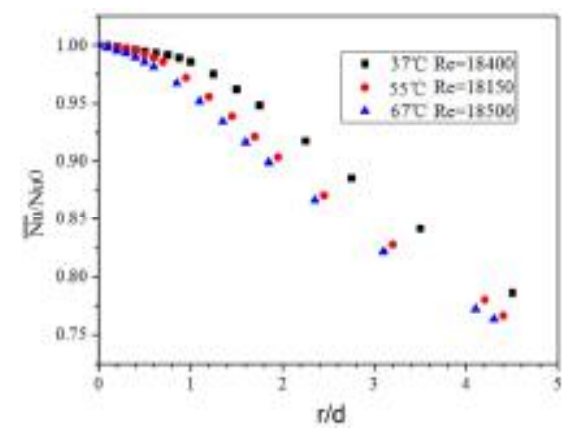

Fig. 6: Radial distribution of average Nu number normalized for mutative temperature

Seen from the Fig. 6, it is clearly observed that the decreasing gap will become obvious due to the growing temperature when the average Nusselt number along the radial distribution is normalized. When the qualitative temperature changes, the physical property of the water will also change. With the reduced temperature, the heat transfer will be enhanced due to the thickened 
thermal boundary layer, the increscent Prandtl number and the increscent viscosity.

\section{Conclusions}

To prepare for making a study on the heat transfer of jet impingement about quaternary mixed nitrate, a new kind of experimental equipment has been established. Meanwhile, in order to ensure the validation of experimental equipment and method, a experimental study on the heat transfer of jet impingement using the water has been carried out in this paper. Some conclusions can be achieved as the following shows.

1、Some fitting correlations have been obtained by utilizing the water in higher temperature, whose results indicate the accuracy and reliability of experimental equipment and method.

2、For the same Reynolds number, the heat transfer effect of water will decrease with the increasing of temperature, which indicates that thermophysical properties of the working fluid have a significant effect on the experimental research.

3. Comparing with the characteristics of heat transfer for different temperature, it is found that the higher temperature make no difference on the stability of this experimental system.

4、 The experimental results in this paper provide a necessary technical guarantee for the study of heat transfer of jet impingement utilizing high temperature molten salt.

\section{Acknowledgements}

This research was sponsored by the National Natural Science Foundation of China (Grant No.51276004) and the National Basic Research Program of China ( "973" Program) (Grant No.2013CB228306).

\section{References}

[1] Liu X, Lienhard V J. Journal of Heat Transfer, 8 (113): 571-582 (1991).

[2] Yaohua Zhao, Chongfang Ma. Journal of Beijing Polytechnic University, 9 (15): 7-13 (1989).

[3] Tie Geng, Dequn Li, Huamin Zhou,Yujie Shao, Weijun Wang. Machinery Design \& Manufacture, 6 (6): 154 -156 (2006).

[4] Jingzhou Zhang, Xiaoming Tan, Bo Liu. Journal of Nanjing University of Aeronautics and Astronautics, 44 (5): 741-746 (2012).

[5] Jinglei Xu, Zhong Xu, Min Xiao, Shujuan Huang. Mechanics in Engineering, 21 (6): 8-17 (1999).

[6] Martin H. Advances in Heat Transfer, 1 (13): 1-60 (1977).

[7] Shiming Yang, Wenquan Tao. Heat Transfer [M]. Beijing: Higher Education Press (2006).

[8] Yuting Wu, Nan Ren, Chongfang Ma. Energy Storage Science and Technology, 2 (6): 586-592 (2013). 